\title{
DATA-DRIVEN DESIGN
}

\author{
Aletta SMITS, Dennis NGUYEN, Erik HEKMAN and Koen VAN TURNHOUT \\ Utrecht University of Applied Sciences, The Netherlands
}

\begin{abstract}
The datafication and digital transformation of society change design professions on a profound level. With data and machine intelligence being the design material of the future, the master's programme Data-Driven Design, developed at HU Utrecht University of Applied Sciences, prepares the next generation of designers to handle the challenges and opportunities that come with this new material. Foundation of the programme is the data-driven feedback loop that serves as a model to aid students in their understanding of what data-driven concepts may accomplish. The programme contains a conceptual track, a human track and a technology track, divided over three domains: Designing for a digital society, Designing for humans and Designing for processes. After teaching the programme for two years, identified challenges in the curriculum were of a didactic nature (how to teach a paradigm such as "critical thinking"), a logistic nature (how to maintain flexibility in the curriculum, but not give up on academic and social integration), and of a content nature (how to maintain a strong connection with the practical field by means of guest speakers, but at the same time provide a coherent narrative for the students). The paper outlines the curriculum design in detail and summarizes how developers and staff addressed the above challenges. Specifically, the challenges we encountered in teaching students to work within the paradigm of critical thinking might be instructive.
\end{abstract}

Keywords: Data-driven design, feedback loop, master, critical thinking, user experience, educational design

\section{INTRODUCTION}

The datafication and digital transformation of society have a profound impact on all design professions. Designers are asked to research, envision, and prototype systems and services in which data and machine intelligence play a vital role. Data and computer intelligence are the design materials of the future [1]. How should the new generation of design professionals be prepared for the challenges and opportunities associated with design in a "data-driven world"? At the HU University of Applied Sciences Utrecht we tackled this question by developing the master's programme Data-driven Design.1 The programme was originally developed for Media Professionals (User Experience Design, Content Design, Service Design, Journalism), but is also open to other bachelor graduates (Computer Science, Marketing, Business). Central to the programme is the idea of the data-driven feedback loop, i.e. that products and services improve simply by being used. In this paper, we provide an overview of our international study programme and discuss some of the challenges that we encountered in designing and teaching this curriculum.

\section{DATA-DRIVEN FEEDBACK LOOP}

The data-driven design loop (see Figure 1) illustrates the essential elements of a data-driven service. Any data-driven service aims to deliver a user experience in a context of use: e.g. a personalized content stream or an adaptive game experience. User actions within such a digital experience yield data which

\footnotetext{
${ }^{1}$ The programme was envisioned by Hans de Clercq, manager of the Institute for Media at HU University of Applied Sciences Utrecht and Hans Bouwknegt, former professor Crossmedia Business. Curriculum and didactic model were developed by Aletta Smits and Erik Hekman. Dennis Nguyen developed the concept track and the graduation track; other courses were developed by Erik Hekman, Bob Cruijsberg, Nathalie Brähler, Daan Oldenhof, Simone de Droog, and Jonas Moons. Project support was provided by Sara Meeder, Tineke Kleene and Sanna Muijlaart.
} 
can subsequently be analysed and lead to adapted user output. A social media service may collect social graph data, games may offer personalized rewards, etc. In each step of the continuous feedback loop, humans and computers work together in a socio-technical system to accomplish the given objectives. User data and adaptive output overlap in Figure 1 since it is exactly in that overlap that the interface is situated: the structure that connects the two.

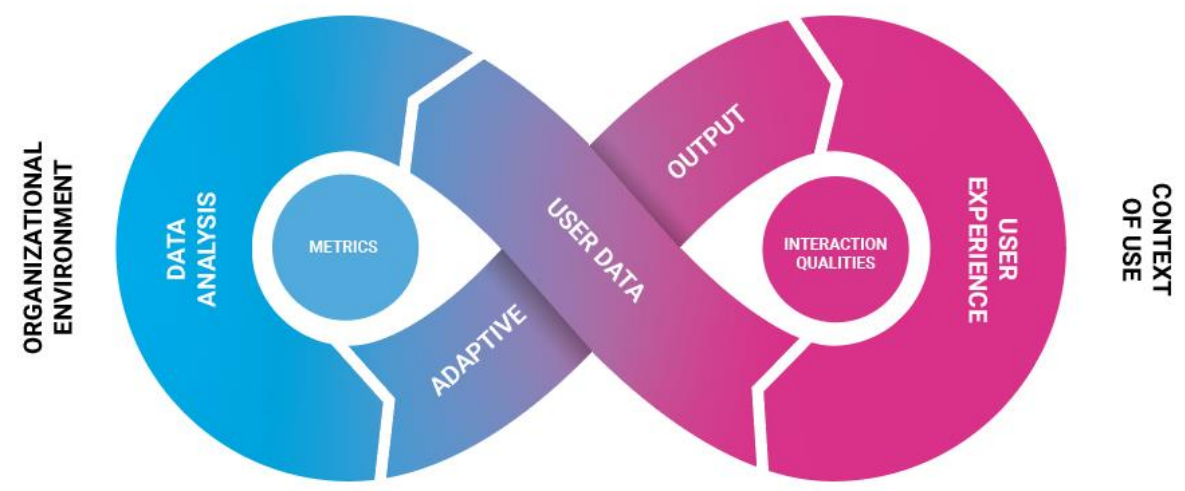

Figure 1. Feedback loop

As the continuous data-feedback loop suggests an optimization process, it is important to identify a focus point for this optimization. On the user side of the loop, the focus is on qualities of the interaction - depicted in the right middle of the loop. Most typically, data-driven services aim for seamless, engaging and/or personal experiences, but these interaction qualities can only be achieved if the data analysis yields reliable information about them. Adaptive games, for example, may try to optimize users' engagement and therefore adjust the difficulty level of the game based on users' behaviour. To do that, metrics place focus on the data analysis on the organizational side of the loop. A data-driven service can only be successful if there is a sound mapping between the interaction qualities, on the one hand, and the metrics, on the other hand. If the metrics give a biased view of the quality of the interaction, the optimization is misaligned. An example for such misalignment is clickbait in content streams. Here, "click-through" is unjustly considered to be an adequate metric of the user's interest in a piece of content (e.g. an article).

Throughout the programme, the data-driven feedback loop serves as the framework that helps students understand the intricacies of data-driven concepts, and that supports operationalization and specification of their ideas. The data-driven design loop served us well as a didactic tool: abstract enough to allow for many different ideas, yet at the same time simple enough for students to easily grasp and use as a tool to for their concept design.

\section{THREE TRACKS IN THREE DOMAINS}

The Data-driven Design programme is structured horizontally and vertically (see Figure 2). Vertically, students work on three different courses, that together form a 10-week narrative. The three sets are labelled domains and are consecutively: Designing for a digital society, Designing for engagement and Designing for process. The names of those domains are purposely active: not just digital society (which would imply 'learning about the digital society'), but the actual designing for a digital society (which demands a far more active mindset and is geared towards application). In each of those blocks, the three courses are in their turn part of a horizontal track: conceptual, technology, or human, integrating the courses throughout the year. The final 10 weeks of the programme cover the end phase, the graduation process: writing a paper that researches a specific data-driven design and building a prototype as a proof of concept. 


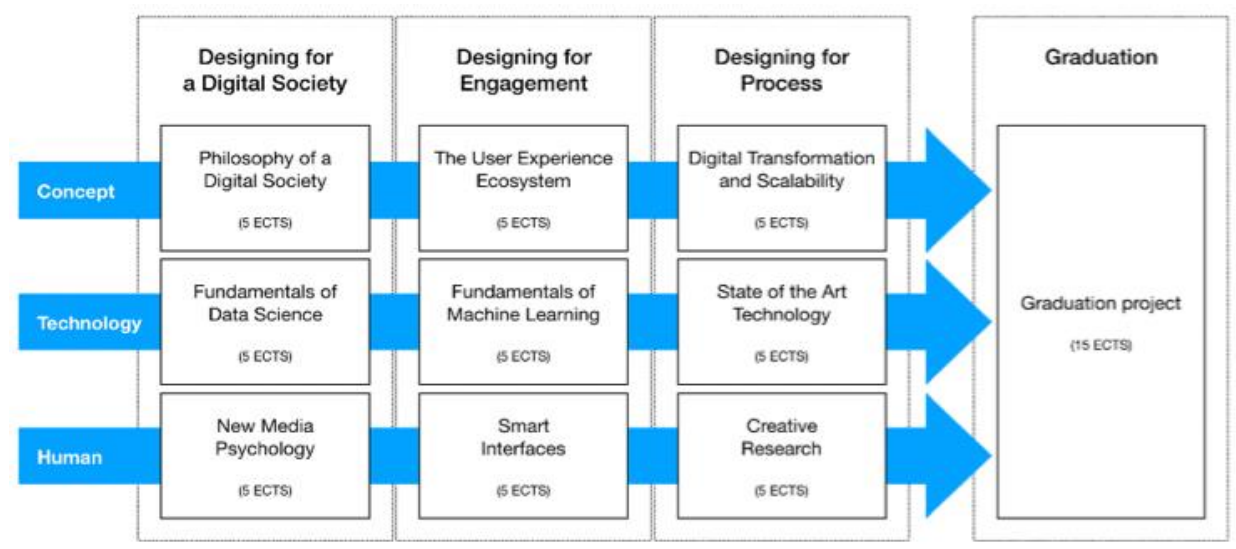

Figure 2. Curriculum Design

\subsection{Conceptual track}

The conceptual track aims at instilling students with an interdisciplinary, critical, and design-oriented perspective on the underlying trends and transformations that shape the digital society. Students are asked to take a broad view and engage with ideas and concepts from a wide range of subjects that address aspects of datafication, digitalization and the subsequent effects on how humans and tech form complex and dynamic networks of interaction. Example questions include: What is a digital society and what are digital cultures? How is media technology constantly changing - but also, what appears to remain (relatively) constant? How does technology change social interactions and trigger emotions? And how can we build innovative businesses and organizations that make ethical use of data and emerging technologies?

The main goals are to 1) provide a deeper understanding of the fundamental transformations that are triggered by digital technologies in business, culture, and society [3][4]; 2) to develop skills in critical thinking and the ability to discuss current issues and potential future developments from an interdisciplinary perspective [2]; 3) to sharpen analytical skills that derive from a holistic view on the mutually influential relationships that emerge from user-tech interaction; 4) to connect all of this to the concrete context of digital transformation, emerging user-experience ecosystems and scalable datadriven business models. This becomes manifest in the three courses that follow in chronological order: A Philosophy for a Digital Society (PDS), The User Experience Ecosystem (UEE), and Digital Transformation \& Scalability (DTS).

Course assignments capture the diversity of content and are connected to different forms of professional, academic, and critical communication; they provide a glimpse at the requirements for the graduation project at the end of the study programme. This includes 1) oral exams in form of interactive debates; 2) academic essays, in which students select and analyse a relevant case that illustrates the complexities of user experience and technology; 3) White papers, in which students propose ideas for data-driven business (or organizational) models that put value-creation for the user at the centre, focus on trust, and explore possibilities for building them with new technologies (e.g. decentralization). In this way students show their skills in translating theoretical knowledge into concrete concepts for practical solutions in a domain of their choice (public or private) [5][6].

Ensuring constant topicality and relevance of examples in the courses is imperative, as is allowing for permanent reflection on those examples and their impact. Simultaneously, it is crucial to connect current cases to established theoretical frameworks, models, and definitions from academic literature.

\subsection{Technology track}

The technology track aims at giving students hands-on experience with working with data while at the same time confronting them with the fact that any choice they make (in interpretation of data, the inclusion or exclusion of data points, the selection of a technology) can have far-reaching consequences for the constructs they are examining. Again, even when the focus in these classes can sometime be on 'getting the code to work', the reflection is a crucial part of these course: what does your code achieve, and is that a desirable effect?

With a mix of students from all walks of life, we have students in class that are comfortable with coding (Java, Python, PHP, C++), and students who have only scant knowledge of HTML and CSS. However, 
even students with a technical background are not necessarily comfortable with data gathering, processing, and analysis (i.e. understanding and using algorithms). We start, therefore, at the beginning of scraping and processing. This is also necessary to help them turn their focus not only on writing the code, but to continue considering what they are achieving with it. This track does not turn our students into fully-fledged data-scientists, therefore, but provides them with enough hands-on experience to be conversational partners for those data scientists, and statisticians, econometrists, software developers, etc.

In the first course, the Fundamentals of Data Science (FDS), students learn how data can be found anywhere in society, how it can be technically collected from open data sources, social media, and web scraping, and subsequently cleaned and processed it in order to produce descriptive analyses of the society they live in. They find a concept in either of the two other courses in the Designing for a digital society-domain, and develop a dashboard that helps explore digital society and digital culture (such as the visualization of hashtags in order to investigate internet activism).

In Fundamentals of Machine Learning $(F M L)$, students use their collected data to start understanding the application of algorithms: clustering, classification, forecasting, recommender systems and text mining. This course focuses, therefore, on learning from the constant feedback loops between human and machine and strongly connects with the concepts covered in the two other course in this domain: Smart Interfaces and the User Experience Ecosystem.

In the final course, State of the Art in Technology (SAT), students expand their technological horizon. The course focuses on new technological innovations and how they can be used to develop new processes and concepts. SAT promotes playing, experimenting, pushing the limits of technology (sensors, image tech, speech technology, gestures, etc.). It does so by adopting a critical making perspective during the development of prototypes. Prototypes are thus not seen as an accurate depiction of a product but to critically reflect, to uncover and alter the limitations of design practice i.e., the technology [7]. This attitude has been promoted all along the course and is essential for our programme: we want to train data-driven designers with a strong ethical perspective and a strong sense of the impact of their creations.

\subsection{Human track}

The human track places the user as a connected individual at the centre of attention. Courses in this track emphasize that interaction with and via digital technology is predominantly a subjective experience. Social, cultural, and psychological factors all shape a use of technology and, therefore, interaction with designs. Understanding these dynamics supports the development of practical solutions that ideally maintain user engagement at a high level.

Central questions in this track are: How do users engage with digital devices and services? What motivations drive them and what influence do social and cultural factors play? How do they adopt technology and how do they experience it? How do users interact with technology via interfaces? And how can we plan and execute applied research projects to build a data-driven prototype that learns from the user and adapts to her needs? Again, three courses address these general learning objectives.

The first, New Media Psychology, introduces theories and models from the intersection of social psychology, communication sciences, and media studies. Key topics are media effects, audience research, biases and heuristics, media consumption, interpersonal communication, social networks/mediated socialization processes, human-computer-interaction, and framing. Secondly, Smart Interfaces focuses on the role of interfaces as the main medium between the human use and the algorithmic, data-driven solution. As such this course is the pivotal point in our data-driven design loop: it connects the user data with the adaptive output. Finally, the course Creative Research prepares students for their graduation project. They work on a research proposal that is both the main assignment for passing the course and the basis for their graduation assignment.

\subsection{Graduation}

For the graduation project, students work on a data-driven solution for a practical problem. It consists of two parts: an academic paper and a prototype. Both have been prepared in the Creative Research course in the previous ten-week block. In the graduation project, students show that they have become confident discourse participants with a sound academic understanding of datafication and the digital transformation but also practice-focused designers of data-driven solutions. The learning goals include: (1) the ability to research, analyse and understand the use of data in specific contexts of use; (2) to 
develop data-driven concepts; (3) to develop and test a data-driven prototype; (4) to critically evaluate the development process and the potential impact of the proposed prototype on the domain in focus; (5) and to communicate this clearly to diverse audiences of relevant stakeholders. The graduation phase hence unites the three tracks of conceptual, human, and tech in one project.

The purpose of the academic paper is to put the project into a research context. For the prototype, we are mainly interested in the critical reflection on the various iterations of the prototype ("critical making") [7] rather than its functionality. For example, rather than giving a sufficient mark for an algorithm that actually clusters entities in a particular way, we are interested in the process that led to that algorithm: what did previous iterations teach the student about clustering? Is this clustering - while it works technically - still desirable? How does it add to the experience? Rather than being overwhelmed by technical features, we would like our students to reflect on what is accomplished by their lines of code. In the context of the graduation project, this critical making approach is an important way to consider the design process and address limitations in design practice. The prototype should, therefore, stimulate discussion on how certain interactions within a specific domain of the digital society change with the introduction of a data-driven solution and what value it could create. Reflection and practical action thus go hand in hand.

In the short time this master has been taught, students have researched e.g. internet activism (within the domain of art and health care), carrying capacity of popular cities (within the domain of tourism) and the Duolingo algorithm in order to enhance retention of facts (within the domain of education).

\section{DISCUSSION OF SOME OF THE OBSTACLES AND SOLUTIONS}

Our objective is to support students' turn into professional discourse partners on digital culture, competent designers that are deeply aware of the impact of data on design, and researchers at a master level that are not only able to write a sound academic paper, using the traditional way of arguing, but also build a series of technical prototypes within the paradigm of critical making. Obviously, in doing so, we encountered challenges. For this paper we highlight two of them:

\subsection{Didactic challenges and changes}

With students coming from all academic and professional backgrounds, with various levels of technical skills, English proficiency, and rigor in academic training, didactic challenges are manifold. We would like to focus on one didactic challenge, however: teaching students to work and think within the paradigm of "critical making" [7]. In the first iteration of the programme, we had worked with critical making, illustrated its purpose, and talked many times about why critical making was more than building a set of prototype iterations but using prototypes to reflect and learn. Still, students were noticeably insecure about their projects: they understood the demands of an academic paper, but they were worried about their prototype and what it exactly was that their examiners were looking for in a series of prototypes within the 'critical making'-paradigm. They focused on the result of the prototype, more than that they would focus on the insights and understanding that each iteration had yielded. Our analysis showed that this was partly since we had intended them to work on the paper and the prototype in parallel, thereby enhancing the quality of both, in a didactic feedback loop. However, the students had overwhelmingly decided to first write their paper and then work on their prototyping. That meant there was less time for iterations, and by having finished their paper, their thinking was to a certain extent 'locked'. Another factor was that during their bachelor programmes, students had been trained in delivering 'complete products' rather than products of thinking: they rather produced a fancy interface than a thorough reflection on their experiments with a particular algorithm. Our solution, for now, was to create the Creative Research course, specifically so they can prepare a graduation proposal, that includes the ideation of the prototyping process. Parallel to the Creative Research course, students take the State of the Art in Technology course, that also challenges them to build prototypes within the paradigm of critical thinking. We are currently investigating if the close integration between those two courses (both content-wise and timewise) will be of assistance in grasping the concept of critical thinking.

\subsection{Connections to the practical field and how we changed it}

Guest lectures and workshops held by external parties are overall crucial for maintaining a strong link to both the practical field and broader academic discourse; it helps validating content and didactics of the programme and connects students (but also teaching staff) to the professional world. However, this 
linkage needs firm embedding in the courses and enough resources to harness the full potential. Originally, we had envisioned a weekly guest speaker that would provide inspiring talks about datadriven design examples out there in real life. However, while the guest lecturers added value to the classroom experience for students, their talks and workshops were, while sensible additions, still not fully integrated elements in the learning process. Students perceived the long string of guests as disruptive and not always an asset. In the second iteration of the programme, therefore, the coordinators decided to maintain the connection with the practical field by organizing well-defined workshops in test labs of our partners and to invite a smaller group of guest speakers and make their contributions integral to the learning process by connecting it to the assignments in the respective courses.

\section{CONCLUSIONS}

Our challenge was to design a curriculum that helped Media Professional understand and use the concepts of data-driven design (feedback loop), think critically about its impact (critical making) and see the creativity in it as well. We attempted to do that by teaching them how to work with technology, but always from the perspective of creativity. We also attempted to do that by empowering them in their discourse with hard-core statisticians and data scientists with arguments and concepts and a sound theoretical foundation, both in datafication as in new media psychology and sociology. We hope this programme contributes to raising a generation of designers that knows how to deal with the material of the future (data) while never losing sight of the society and the people they design for.

\section{REFERENCES}

[1] Holmquist, L. E. Intelligence on tap: artificial intelligence as a new design material. Interactions, 2017, 24(4), 28-33.

[2] Boyd, D. and Crawford, K. (2012): 'Critical Questions for Big Data', in Information, Communication \& Society15 (5), pp. 662-679.

[3] Kitchin, R. (2014): The Data Revolution. Big Data, Open Data, Data Infrastructures and Their Consequences. Sage: London.

[4] Loukissas, Y. A. (2019): All Data are Local. Thinking Critically in A Data-Driven Society. Cambridge: MIT Press.

[5] Maciejewski, M. (2017): 'To Do More, Better, Faster and More Cheaply. Using Big Data in Public Administration', in International Review of Administrative Sciences83 (15), pp. 120-135.

[6] McAfee, A. and Brynjolfsson, E. (2017): Machine Platform Crowd. Harnessing our Digital Future. Norton: New York

[7] Sengers, P., Boehner, K., David, S., \& Kaye, J. J. (2005, August). Reflective design. In Proceedings of the $4^{\text {th }}$ decennial conference on Critical computing: between sense and sensibility (pp. 49-58).

[8] Braxton, J. M., Doyle, W. R., Hartley III, H. V., Hirschy, A. S., Jones, W. A., \& McLendon, M. K. (2013). Rethinking college student retention. John Wiley \& Sons. 